\title{
Self-similarity in the description of inclusive spectra of the hadrons produced in pp- and AA-collisions
}

\author{
A.Malakhov ${ }^{1}$ \\ Veksler and Baldin Laboratory of High Energy Physics, JINR \\ 141980, JINR, Dubna, Russia \\ E-mail:malakhov@lhe.jinr.ru

\section{G.Lykasov} \\ Dzhelepov Laboratory of Nuclear Problems, JINR \\ 1419080, JINR,Dubna, Russia \\ E-mail: lykasov@jinr.ru
}

The use of the self-similarity allows us to describe rather well the ratio of the proton to anti-proton yields in A-A collisions as a function of the energy in a wide range from 10-20 GeV to a few TeV. Modification of our approach taking into account the energy dependence of inclusive spectra of hadrons allows us to describe the differential cross section as a function of $\mathrm{m}_{\mathrm{T}}$. We have got a satisfactory description of the slopes of the hadron spectra as functions of the energy in a wide range from AGS to LHC.

XXII International Baldin Seminar on High Energy Physics Problems

15-20 September 2014

JINR, Dubna, Russia

${ }^{1}$ Speaker

(c) Copyright owned by the author(s) under the terms of the Creative Commons Attribution-NonCommercial-ShareAlike Licence. 


\section{Introduction}

In the 70-ies of the previous century by the initiative of academician A.M. Baldin in Dubna at the Laboratory of High Energy Physics (LHE) of the Joint Institute for Nuclear Research (JINR) a new research direction - relativistic nuclear physics, - was founded. This was, first of all, conditioned by accelerating the relativistic deuterons at the energy of $4.5 \mathrm{GeV} /$ nucleon at the Synchrophasotron of the LHE, where academician A. M. Baldin was the director at that time. In addition, A. M. Baldin in [1] made a prediction of the so-called nuclear cumulative effect, subsequently discovered at the Synchrophasotron by the group of professor V.S. Stavinskiy [2]. All this allowed one to begin intensive studies of relativistic nucleus interactions at energies up to 4.5 $\mathrm{GeV} /$ nucleon. Later A.M. Baldin was invited to consider relativistic nuclear interactions in the four dimension velocity space [3].

The approach of studying relativistic nuclear interactions in the four dimension velocity space proved to be very fruitful [4]. This article presents further development of this approach.

\section{The parameter of self-similarity}

In their time together with A. M. Baldin we published an article [5] with the predictions of the ratios of the particle outputs in the collisions of nuclei at high energies.

Let us put down the interaction of nucleus I with nucleus II resulting in the inclusive particle 1 production as follows:

$$
\mathrm{I}+\mathrm{II} \rightarrow 1+\ldots
$$

According to Baldin's assumption more than one nucleon in the nucleus I can participate in the interaction (1). The value of $\mathrm{N}_{I}=\lambda \cdot \mathrm{A}_{\mathrm{I}}$ is the efficient number of nucleons inside the nucleus I, participating in the interaction which is called the cumulative number. Its values lie in the region of $0 \leq N_{I} \leq A_{I}$. The cumulative area complies with $\mathrm{N}_{\mathrm{I}}>1$. Of course, the same situation will be for the nucleus II, and you can enter the cumulative number of $\mathrm{N}_{\text {II }}$.

For reaction (1) with the production of the inclusive particle 1, we can write the law of conservation of four-momentum in the following form:

$$
\left(\mathrm{N}_{\mathrm{I}} \cdot \mathrm{P}_{\mathrm{I}}+\mathrm{N}_{\mathrm{II}} \cdot \mathrm{P}_{\mathrm{II}}-\mathrm{p}_{1}\right)^{2}=\left(\mathrm{N}_{\mathrm{I}} \cdot \mathrm{m}_{0}+\mathrm{N}_{\mathrm{II}} \cdot \mathrm{m}_{0}+\mathrm{M}\right)^{2} \text {, }
$$

where $\mathrm{N}_{\mathrm{I}}$ and $\mathrm{N}_{\mathrm{II}}$ are cumulative numbers (the number of nucleons involved in the interaction); $\mathrm{P}_{\mathrm{I}}, \mathrm{P}_{\mathrm{II}}, \mathrm{p}_{1}$ are four momenta of the nuclei I and II and particle 1, respectively; $\mathrm{m}_{0}$ is the mass of the nucleon; $\mathrm{M}$ is the mass of the particle providing the conservation of the baryon number, strangeness, and other quantum numbers.

In [6] the parameter of self-similarity is introduced, which allowed one to describe the differential cross section of the yield of a large class of particles in relativistic nuclear collisions: 


$$
\Pi=\min \left[1 / 2\left[\left(\mathrm{u}_{\mathrm{I}} \cdot \mathrm{N}_{\mathrm{I}}+\mathrm{u}_{\mathrm{II}} \mathrm{N}_{\mathrm{II}}\right)^{2}\right]^{1 / 2}\right.
$$

where $u_{I}$ and $u_{I I}$ are four velocities of the nuclei I and II.

In this case the invariant cross-sections of the output inclusive particles of different types at nucleus interactions with atomic numbers $A_{I}$ and $A_{I I}$, are described by the universal dependence in a wide energy range and different atomic numbers of the colliding nuclei:

$$
\mathrm{E} \cdot \mathrm{d}^{3} \sigma / \mathrm{dp}^{3}=\mathrm{C}_{1} \cdot \mathrm{A}_{\mathrm{I}}^{\alpha\left(\mathrm{N}_{\mathrm{I}}\right)} \cdot \mathrm{A}_{\mathrm{II}}^{\alpha\left(\mathrm{N}_{\mathrm{II}}\right)} \cdot \exp \left(-\Pi / \mathrm{C}_{2}\right),
$$

where $\alpha\left(\mathrm{N}_{\mathrm{I}}\right)=1 / 3+\mathrm{N}_{\mathrm{I}} / 3, \quad \alpha\left(\mathrm{N}_{\mathrm{II}}\right)=1 / 3+\mathrm{N}_{\mathrm{II}} / 3$,

$\mathrm{C}_{1}=1.9 \cdot 10^{4} \mathrm{mb} \cdot \mathrm{GeV}^{-2} \cdot \mathrm{c}^{3} \cdot \mathrm{st}^{-1}$ и $\mathrm{C}_{2}=0.1250 .002$.

\section{Self-similarity parameter in the central rapidity region}

In the central rapidity region it is possible to find the analytical expression for $\Pi$ [5]. For this case $\mathrm{N}_{\mathrm{I}}$ and $\mathrm{N}_{\text {II }}$ are equal to each other: $\mathrm{N}_{\mathrm{I}}=\mathrm{N}_{\mathrm{II}}=\mathrm{N}$.

where

$$
\mathrm{N}=\left[1+\left(1+\Phi_{\delta} / \Phi^{2}\right)^{1 / 2}\right] \cdot \Phi
$$

$$
\begin{aligned}
& \Phi=2 \mathrm{~m}_{0} \cdot\left(\mathrm{m}_{\mathrm{T}} \mathrm{ch} \mathrm{Y}+\mathrm{M}\right) / \mathrm{sh}^{2} \mathrm{Y} \\
& \Phi_{\delta}=\left(\mathrm{M}^{2}-\mathrm{m}_{1}{ }^{2}\right) /\left(4 \mathrm{~m}_{0}{ }^{2} \cdot \mathrm{sh}^{2} \mathrm{Y}\right) .
\end{aligned}
$$

Here $\mathrm{m}_{1 \mathrm{~T}}$ is the transverse mass of the particle $1, \mathrm{~m}_{1 \mathrm{~T}}=\left(\mathrm{m}_{1}{ }^{2}+\mathrm{p}_{\mathrm{T}}{ }^{2}\right)^{1 / 2}$. And then -

$$
\Pi=\mathrm{N} \cdot \operatorname{chY}
$$

From equation (4) it follows that the ratio of cross sections (R) of process 1 to process 2 is as follows:

For baryons we have

$$
\mathrm{R}=\exp \left[-\left(\Pi_{1}-\Pi_{2}\right) / \mathrm{C}_{2}\right]
$$

and for antibaryons -

$$
\Pi_{\mathrm{b}}=\left(\mathrm{m}_{1 \mathrm{~T}} \cdot \operatorname{chY}-\mathrm{m}_{1}\right) \cdot \operatorname{chY} /\left(\mathrm{m}_{0} \cdot \operatorname{sh}^{2} \mathrm{Y}\right)
$$

$$
\Pi_{\mathrm{a}}=\left(\mathrm{m}_{1 \mathrm{~T}} \cdot \operatorname{ch} \mathrm{Y}+\mathrm{m}_{1}\right) \cdot \operatorname{chY} /\left(\mathrm{m}_{0} \cdot \mathrm{sh}^{2} \mathrm{Y}\right) .
$$

The difference $\Pi_{\mathrm{b}}-\Pi_{\mathrm{a}}$ will not depend on $\mathrm{m}_{1 \mathrm{~T}}$ :

$$
\Pi_{\mathrm{b}}-\Pi_{\mathrm{a}}=-2 \mathrm{~m}_{1} \cdot \mathrm{chY} /\left(\mathrm{m}_{0} \cdot \mathrm{sh}^{2} \mathrm{Y}\right) .
$$

The results of calculations for the ratio of the antiproton cross section to the proton one by using (12) and (9) is presented in Fig. 1 together with the experimental data including the latest data at LHC [7-9]. 


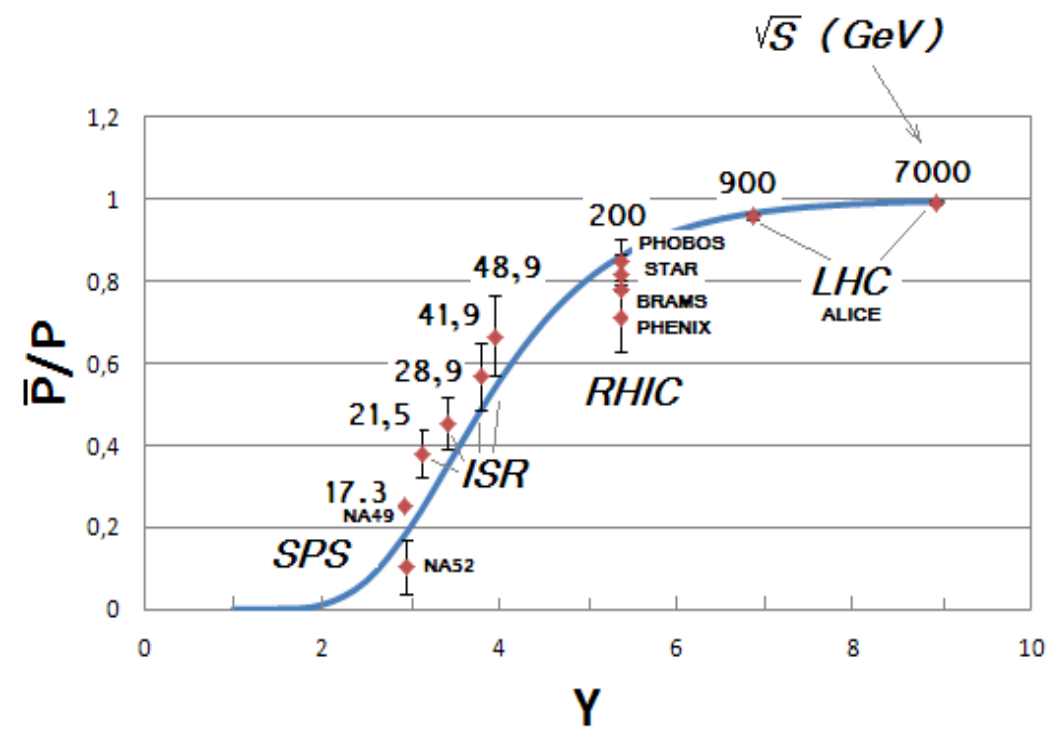

Fig.1. Dependence of the ratio of the antiproton cross section to the proton one on the rapidity and energy of the interacting nuclei.

The same calculations can be made for other antiparticles and particles (Fig.2). However, there are poor experimental data in the central rapidity region.

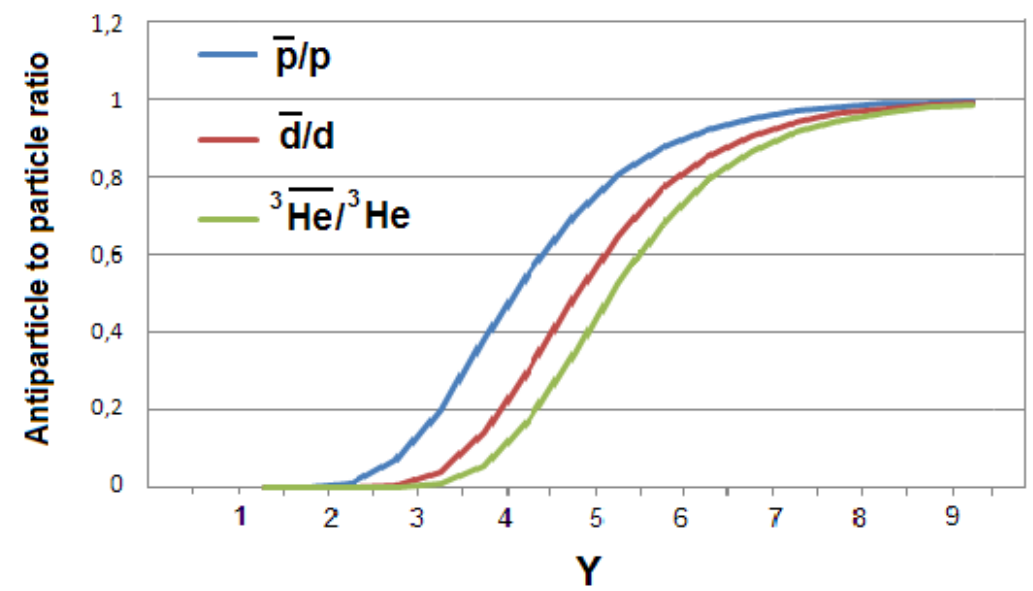

Fig.2. Results of calculations of the dependence of the antiproton to proton, antideuteron to deuteron and antihelium-3 to helium-3 cross section ratios on the rapidity of interacting nuclei in the central rapidity region.

\section{Further development of Baldin's approach}

For the inclusive cross-section of hadron production in the central rapidity region and according to AGK (Abramovsky, Gribov, Kanchelly) cancellation [10] we can write the following expression for the quark contribution:

$$
\mathrm{E}\left(\mathrm{d}^{3} \sigma / \mathrm{dp}^{3}\right)_{\mathrm{q}}=\varphi_{\mathrm{q}}\left(\mathrm{y}=0, \mathrm{p}_{\mathrm{t}}\right) \cdot \Sigma\left[\mathrm{n} \cdot \sigma_{\mathrm{n}}(\mathrm{s})\right] .
$$

According to [11] we write: 


$$
\mathrm{E}\left(\mathrm{d}^{3} \sigma / \mathrm{dp}^{3}\right)_{\mathrm{q}}=\varphi_{\mathrm{q}}\left(\mathrm{y}=0, \mathrm{p}_{\mathrm{t}}\right) \cdot \Sigma\left[\mathrm{n} \cdot \sigma_{\mathrm{n}}(\mathrm{s})\right]=\varphi_{\mathrm{q}}\left(\mathrm{y}=0, \mathrm{p}_{\mathrm{t}}\right) \cdot \mathrm{g}\left(\mathrm{s} / \mathrm{s}_{0}\right)^{\Delta} .
$$

According to $[12,13]$ the inclusive cross-section of hadron production, taking into account the gluon contribution, can be written in the following form:

$$
\begin{gathered}
\mathrm{E}\left(\mathrm{d}^{3} \sigma / \mathrm{dp}^{3}\right)_{\mathrm{g}}=\varphi_{\mathrm{g}}\left(\mathrm{y}=0, \mathrm{p}_{\mathrm{t}}\right) \cdot \Sigma\left[\mathrm{n} \cdot \sigma_{\mathrm{n}}(\mathrm{s})\right]=\varphi_{\mathrm{q}}\left(\mathrm{y}=0, \mathrm{p}_{\mathrm{t}}\right) \cdot \Sigma\left[(\mathrm{n}-1) \cdot \sigma_{\mathrm{n}}(\mathrm{s})\right]= \\
=\varphi_{\mathrm{g}}\left(\mathrm{y}=0, \mathrm{p}_{\mathrm{t}}\right) \cdot\left[\mathrm{g}\left(\mathrm{s} / \mathrm{s}_{0}\right)^{\Delta}-\sigma_{\mathrm{nd}}\right] .
\end{gathered}
$$

Thus, taking into account the quark and gluon contributions we will receive the following expression:

$$
\mathrm{E}\left(\mathrm{d}^{3} \sigma / \mathrm{dp} \mathrm{p}^{3}\right)=\left[\varphi_{\mathrm{q}}\left(\mathrm{y}=0, \mathrm{p}_{\mathrm{t}}\right)+\varphi_{\mathrm{g}}\left(\mathrm{y}=0, \mathrm{p}_{\mathrm{t}}\right) \cdot\left(1-\sigma_{\mathrm{nd}} / \mathrm{g}\left(\mathrm{s} / \mathrm{s}_{0}\right)^{\Delta}\right)\right] \cdot \mathrm{g}\left(\mathrm{s} / \mathrm{s}_{0}\right)^{\Delta} .
$$

In formulas $(13-16)$ we apply the following symbols:

$\sigma_{\mathrm{n}}-$ cross-section of hadron production by means of the n-pomeron exchange;

$\varphi=\varphi(\Pi)$;

$\mathrm{g}$ - constant ( $\sim 20$ mbarn), which is calculated within the "quasi-eikonal" approximation [11];

$\mathrm{S}_{0}=1 \mathrm{GeV}^{2}$

$\Delta=\left[\alpha_{\mathrm{p}}(0)-1\right] \sim 0,08$, where $\alpha_{\mathrm{p}}(0)$ is the subcritical Pomeron intercept.

Using (16) we can calculate the inclusive cross section of hadron production as a function of the transverse mass.

The results of these calculations are shown in Fig.3. The results normalized on the data of the NA61 experiment at the energy of $31 \mathrm{GeV}$ and multiplied by factor $\left(\mathrm{s} / \mathrm{s}_{0}\right)^{\Delta}$. In the calculations we used the value of constant $C_{2}=0.1816$ (instead of 0.125 ), since it gives the best agreement with the experiment.

One can see a good agreement of the calculated results with the experimental data in a wide energy range from 31 to $7000 \mathrm{GeV}$.

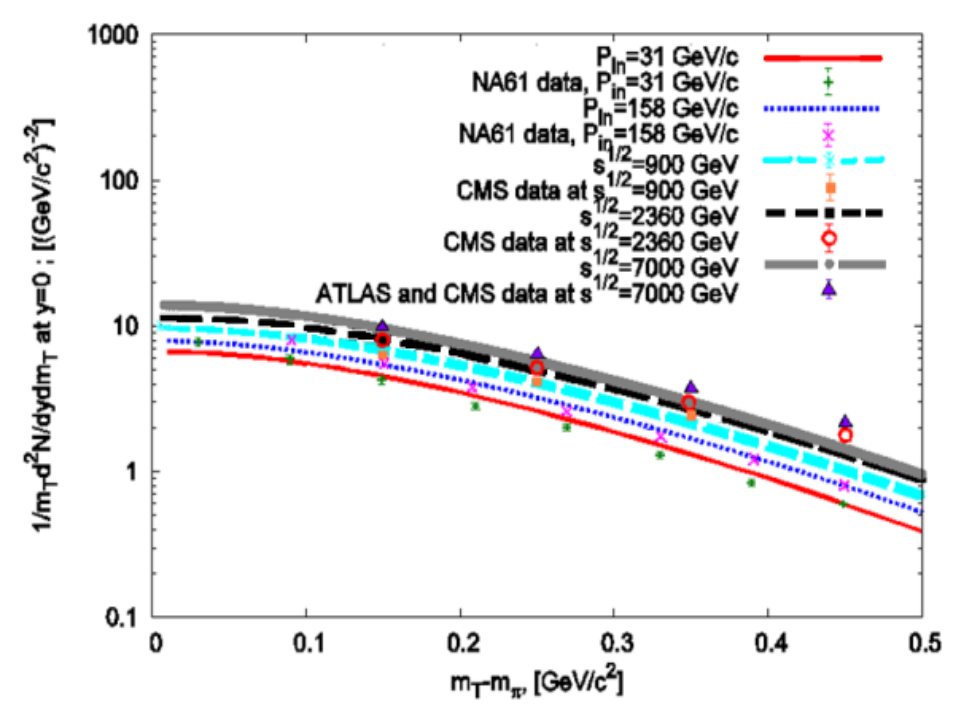

Fig.3. Results of the calculations of the inclusive cross section of hadron production as a function of the transverse mass. The results normalized on the data of the NA61 experiment at the energy of $31 \mathrm{GeV}$ and multiplied by factor $\left(\mathrm{s} / \mathrm{s}_{0}\right)^{\Delta}$. 
It turned out that with constant $\mathrm{C}_{2}=0.1816$ it is better to describe the ratio of the outputs of antiprotons to protons (Fig. 4).

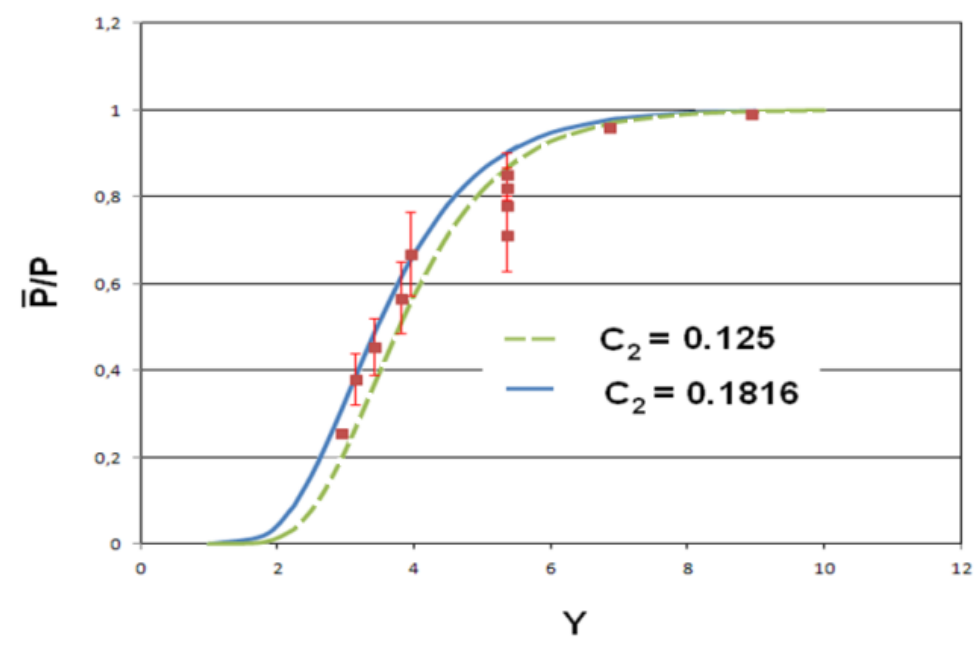

Fig.4. Dependence of the antiproton to proton cross section ratios on the rapidity of interacting nuclei with $\mathrm{C}_{2}=0.125$ and $\mathrm{C}_{2}=0.1816$.

Using the approach described above we have also performed the calculations of the inverse slope parameter $\mathrm{T}$ on the energy dependence for the negative pion production in pp- interactions (Fig.5). There is a good agreement with the experimental data [14].

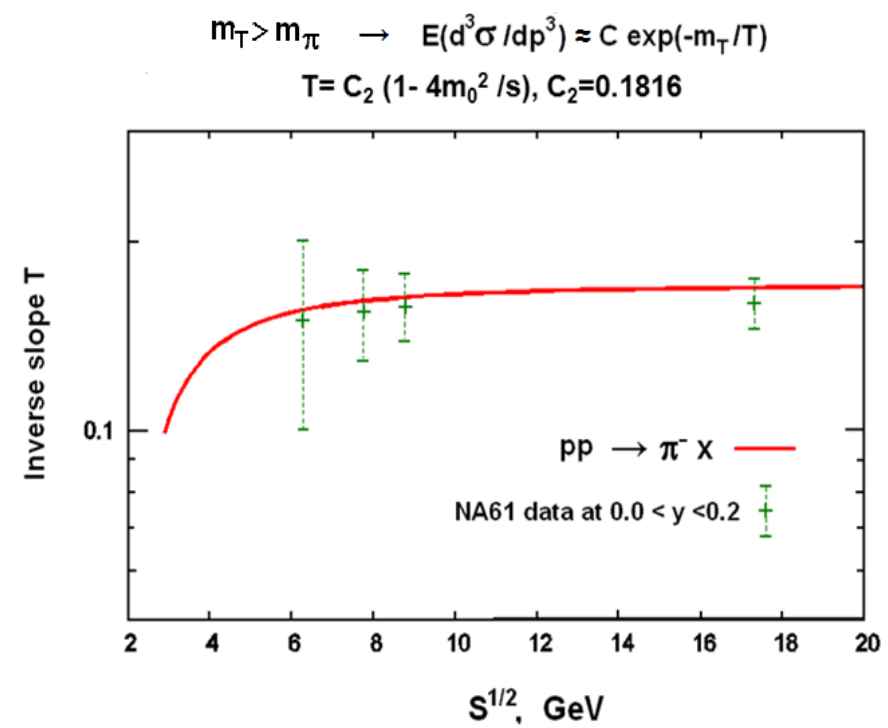

Fig.5. Results of calculations of the inverse slope parameter $T$ on the energy dependence for the negative pion production in pp-interactions. The experimental points are taken from [14]. 


\section{Conclusion}

The use of the self-similarity parameter allows one to rather well describe the ratio of the proton to anti-proton yields in A-A collisions as a function of the energy in a wide energy range from $10 \mathrm{GeV}$ to a few $\mathrm{TeV}$. To study the similar ratio of light nuclei to anti-nuclei, we need more detailed experimental data.

The inclusive spectra in the space of four-velocities depend on the relativistic invariant variables and initial energy. Modification of our approach taking into account the energy dependence of inclusive spectra of hadrons has allowed us to describe the differential cross section as a function of $\mathrm{m}_{\mathrm{T}}$. We have got a satisfactory description of the slopes of the hadron spectra as functions of the energy in a wide energy range of AGS and LHC.

\section{References}

[1] А.М.Балдин. Краткие сообщения по физике ФИАН, №1, 35, (1971).

[2] А.М.Балдин и др. Ядерная физика, т.18, вып.1, 79 (1973).

[3] A.M.Baldin, L.A.Didenko. Fortsch.Phys. 38 (1990) 261-332.

[4] A.M.Baldin, A.I.Malakhov, and A.N.Sissakian. Physics of Particles and Nuclei, Vol.32. Suppl. 1, 2001, pp.S4-S30.

[5] A.M.Baldin, A.I.Malakhov. JINR Rapid Communications, No.1 (87)-98, 5-12 (1998).

[6] A.M.Baldin, A.A.Baldin. Phys. Particles and Nuclei, v.29, No.3, 232 (1998).

[7] A. Tawfik. Nuclear Physics A 859, 63-72 (2011).

[8] http://hepdata.cedar.ac.uk/view/p7907.

[9] R. Klingenberg et al. Nuclear Physics A 610, 306c-316c (1996).

[10] V.Abramovsky, V.N.Gribov and O.Konchelli, Sov.J.Nucl.Phys. 18, 308 (1973).

[11] K.A.Ter-Martirosyan. Sov.J.Nucl.Phys., 44, 817 (1986).

[12] V.A.Bednyakov, A.A.Grinyuk, G.I.Lykasov, M.Pogosyan. Int.J.Mod.Phys., A27

(2012) 1250042.

[13] A.A.Grinyuk, G.I.Lykasov, A.V.Lipatov, N.P.Zotov. Phys.Rev.D87 (2013) 074017.

[14] A.A.Abgrall et al. Eur.Phys.J., C74 (2014) 2794. 